\title{
Experimental study of breakdown voltage and effective secondary electron emission coefficient for a micro-plasma device
}

Mariotti, D., McLaughlin, JAD., \& Maguire, PD. (2004). Experimental study of breakdown voltage and effective secondary electron emission coefficient for a micro-plasma device. Plasma Sources Science \& Technology, 13(2), 207-212. https://doi.org/10.1088/0963-0252/13/2/003

Link to publication record in Ulster University Research Portal

\section{Published in:}

Plasma Sources Science \& Technology

Publication Status:

Published (in print/issue): 01/05/2004

DOI:

10.1088/0963-0252/13/2/003

\section{Document Version}

Publisher's PDF, also known as Version of record

\section{General rights}

Copyright for the publications made accessible via Ulster University's Research Portal is retained by the author(s) and / or other copyright owners and it is a condition of accessing these publications that users recognise and abide by the legal requirements associated with these rights.

\section{Take down policy}

The Research Portal is Ulster University's institutional repository that provides access to Ulster's research outputs. Every effort has been made to ensure that content in the Research Portal does not infringe any person's rights, or applicable UK laws. If you discover content in the Research Portal that you believe breaches copyright or violates any law, please contact pure-support@ulster.ac.uk. 


\title{
Experimental study of breakdown voltage and effective secondary electron emission coefficient for a micro-plasma device
}

\author{
D Mariotti ${ }^{1}$, J A McLaughlin and P Maguire \\ NIBEC, University of Ulster at Jordanstown, Newtonabbey BT37 0QB, UK \\ E-mail: davide@std-ltd.com
}

Received 2 July 2003

Published 27 February 2004

Online at stacks.iop.org/PSST/13/207 (DOI: 10.1088/0963-0252/13/2/003)

\begin{abstract}
A micro-plasma device is used as a gas sensor. The micro-plasma device produces dc discharges with a parallel-plane electrode configuration. In this paper the breakdown voltage is measured for argon with tin oxide electrodes, for three different electrode distances $(0.01 \mathrm{~cm}, 0.025 \mathrm{~cm}$ and $0.05 \mathrm{~cm})$ and for pressure between $1.3 \mathrm{kPa}$ and $13.3 \mathrm{kPa}$. The effective secondary electron emission coefficient is then determined from breakdown voltage results; its behaviour is analysed and compared with other experimental results.

Differences between experimental breakdown voltage and a widely accepted analytical form of the breakdown law are also shown and the influence of the secondary electron emission coefficient is underlined.
\end{abstract}

\section{Introduction}

There is wide interest in dc discharges as they find application in several different fields, e.g. thin film processing, plasma display, laser, switches, etc. Of particular importance are micro-size plasma discharges, which present characteristic features and for which scaling rules do not always apply (Auday et al 1998).

Electrodes used in plasma discharges assume particular significance, and it is therefore important to have as much information as possible on their influential behaviour (Jacobs and La Roque 1947, Phelps and Petrovic 1999). Electrode materials such as tin oxide, which has been used for the results in this paper, are of particular interest due to their characteristic optical properties and their wide application in plasma displays.

The breakdown voltage is an essential experimental parameter that is very useful for understanding microplasma discharges. It provides the essential information that somehow separates the gas state from the plasma discharge state. Such measurements also determine another very important parameter, the secondary electron emission coefficient. Secondary electron emission data for various electrode materials and within different plasma discharges are widely discussed in the literature as they can significantly

1 Author to whom any correspondence should be addressed. contribute to plasma modelling (Phelps et al 1993, 1999, Petrovic and Phelps 1997, Phelps and Petrovic 1999, Auday et al 2000, Novak and Bartnikas 2000).

Our overall objective was to develop a micro-plasma device in order to assess the potential for application in gas analysis by use of plasma emission spectroscopy (Hyland et al 2000, Mariotti et al 2000). The design of the micro-plasma device used to produce micro-discharges was developed with particular attention to plasma display technology. For instance, tin oxide offered the necessary electrode transparency required to acquire the light emission from the plasma. The aim was a design in which each micro-plasma device could be used multiple times before being discarded. A matrix configuration such as the one used in plasma displays, would have allowed the activation of pixels separately, each one for a different analysis (Auday et al 2000). On the other hand, this micro-plasma device did not have stringent requirements such as those for plasma displays (e.g. pixel resolution), but it had to deal with plasma formed from different gas components.

Before utilization in the specific application of gas analysis, the electrical characteristics and emission properties of the micro-plasma device were studied with argon. Breakdown measurements and evaluation of the secondary electron emission coefficient were a part of this characterization study. Although the final application of this device is quite different from others found in the literature, and despite the device 
complex geometry, each single pixel can be classified as a dc parallel-plane electrode configuration.

Section 2 will describe in more detail the experimental set-up and the micro-plasma device. This will be followed by a description of the procedure that was followed to obtain the breakdown measurements. In section 3 , breakdown voltage measurements are reported for a range of the product of pressure and distance $(p d)$. The discussion will then lead to the evaluation of the secondary electron emission coefficients. At the end of section 3, a comparison with a well-known breakdown law is carried out, with some comments on the effect of the secondary electron emission coefficient when the latter shows a marked increasing linear trend. Section 4 concludes and summarizes the results of this paper.

\section{Experimental details}

\subsection{Experimental set-up}

Micro-plasma devices were manufactured and used for experiments with a custom power supply unit (Mariotti et al 2000). The power supply unit was complemented with equipment for measurement of electrical parameters.

A micro-plasma device itself is a small glass vessel sealed with epoxy. Two holes in the glass serve as the gas inlet and outlet. The experimental configuration of the micro-plasma device is best seen in figure 1, which depicts the micro-plasma device before assembling.

Two glass substrates with four patterned electrodes each are placed one on top of the other and are kept separated by a square frame of polytetrafluoroethylene (PTFE) film spacer. The three components are brought together and clamped before being sealed with epoxy. This matrix arrangement forms sixteen pixels where electrodes cross each other, with a gap distance determined by the spacer thickness.

The glass substrates are obtained from commercial $k$-glass (Pilkington), which has, on one of the sides, a conducting fluorine doped $\mathrm{SnO}_{2}$ thin film coating (about $250 \mathrm{~nm}$ thick). From here on, this composed thin film will be referred to as the tin oxide layer. Electrodes were obtained by etching the tin oxide according to the desired pattern (as shown in figure 1). The tin oxide coating had a measured resisitivity of about $4.7 \times 10^{-6} \Omega \mathrm{m}$. Considering that after breakdown the current

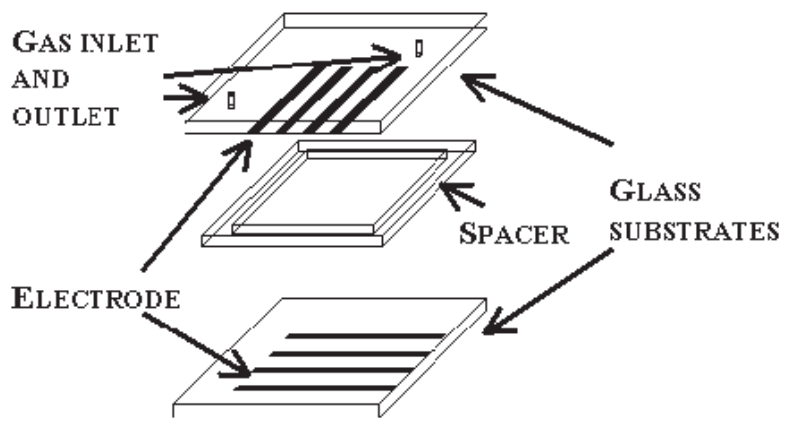

Figure 1. Micro-plasma device before being assembled; the glass substrates are on top and bottom of the drawing and the polytetrafluoroethylene (PTFE) film spacer is in between. Both glass substrates have four metallic electrodes. The top glass substrate has also gas inlet and outlet. measured was in the range of $10^{-2} \mathrm{~mA}$, it is believed that the tin oxide resistivity did not considerably affect breakdown measurements. In addition, plasma discharges produced at these conditions were diffuse over the whole electrode surface without presenting asymmetry with respect to the electrode geometry.

Despite the more complex configuration of the microplasma device as a whole, each pixel presents a simple parallelplane configuration. The geometry and the plasma volume are therefore defined by the electrode width $(0.3 \mathrm{~cm})$ and the spacer thickness $\left(S_{\mathrm{T}}\right)$, i.e. $0.3 \mathrm{~cm} \times 0.3 \mathrm{~cm} \times S_{\mathrm{T}}$. Each pixel was individually activated by selecting an electrode pair, and the breakdown voltage could be measured for each pixel, one at a time.

A laboratory built power supply and measurement system provided a dc voltage ranging from 0 to $600 \mathrm{~V}$. The voltage could be applied individually to each of the sixteen pixels by selection of the appropriate electrode pair. The system could measure several electrical parameters, i.e. the actual applied voltage, the electrode voltage and the current flowing through the electrodes and the generated plasma. Figure 2 depicts the equivalent circuit diagram that has been used for the measurement of breakdown voltage reported in this paper. The discharge current is calculated from the measured voltage over the $1 \mathrm{k} \Omega$ cathode resistor (figure 2). The diagram also shows the sequence of 16 pixels included in the micro-plasma device (dashed outlined box in figure 2). Within the measuring circuitry, a limiting resistor is in series with the micro-plasma discharge at the anode side. The limiting resistor is necessary to limit the current once the discharge has been generated (Jelenak et al 1993, Miller 1964). The limiting resistor value can be selected between 0.068 and $10.068 \mathrm{M} \Omega$, although it has been kept at a value of $3.568 \mathrm{M} \Omega$ for the results reported in this paper.

\subsection{Experimental procedure}

Three micro-plasma devices have been built using different spacing: $0.01,0.025$ and $0.05 \mathrm{~cm}$. This allowed performing breakdown measurements at the three different electrode gaps. For each device, each pixel has been used for a maximum of two breakdown measurements. This is because the final application of the micro-plasma device was in gas analysis, and each pixel was meant to be used in a disposable manner due to

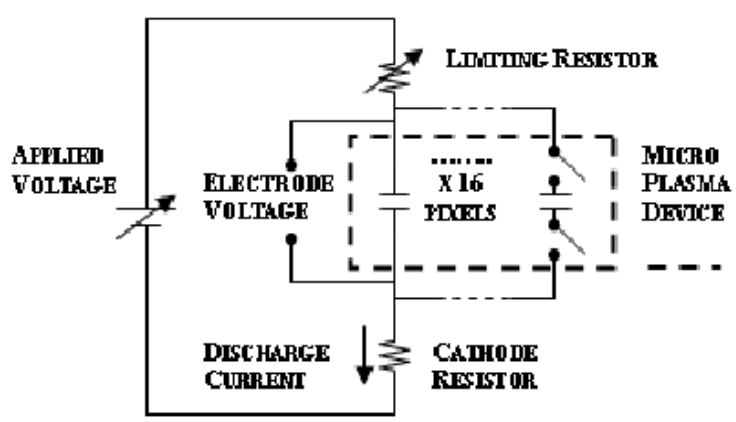

Figure 2. Circuit diagram of the experimental set-up used for breakdown measurements reported in this paper. The broken and thick box outlines the micro-plasma device which contains sixteen different pixels (capacitor-like symbols). Only one pixel at a time is connected to the power supply and measurement system. 
its degradation; i.e. each pixel was intended to be used for very few and short-lived discharges (Auday et al 2000, Lemoine et al 2001). Breakdown measurements were performed for five values of the product, $p d(67,83,100,117$ and $133 \mathrm{~Pa} \mathrm{~cm})$. This means that the argon pressure varied from 6.7 to $13.3 \mathrm{kPa}$ for the micro-plasma device with $0.01 \mathrm{~cm}$ gap distance, from 2.7 to $5.3 \mathrm{kPa}$ for the $0.025 \mathrm{~cm}$ gap one and from 1.3 to $2.7 \mathrm{kPa}$ for the $0.05 \mathrm{~cm}$ one.

After being assembled and sealed with epoxy, the devices were left to dry and therefore electrodes were exposed to ambient air. Prior to breakdown measurements, the devices were evacuated down to a pressure of $1 \mathrm{~Pa}$ for about an hour; subsequently the set point pressure was reached and maintained by controlling argon gas flow into the device. The gas flow was then kept constant during breakdown measurements. No particular attention was devoted to the cleanliness of the electrode surfaces because the final application of the micro-plasma device would not allow an accurate cleaning procedure (Petrovic and Phelps 1997). Therefore, the electrodes should be considered as being dirty surfaces (Phelps and Petrovic 1999).

Breakdown measurements were taken with the device in the dark. A pixel at a time could then be selected and the breakdown voltage was measured by increasing the applied voltage from $200 \mathrm{~V}$ in steps of $2 \mathrm{~V}$. An interval of about a minute between each voltage step was allowed, until the reading of the applied voltage reached stability around the voltage set point. For each measurement, after breakdown occurred, the voltage was increased for a few other voltage steps until the current reached stable values in the normal discharge regime (below $0.03 \mathrm{~mA}$ )

\section{Results and discussion}

\subsection{Breakdown voltage}

Results of the breakdown measurements are shown in figure 3; each data point represents the mean of five or six measurements with different pixels but the same device. The corresponding standard deviations are also shown (figure 3). Three different curves can be observed for the three gap distances, and the standard deviations confirm the three distinct behaviours for the three sets of measurements. All three curves seem to have very similar trend and reach very close voltages for low $p d$ values. Also, the minimum for each curve lies in the same

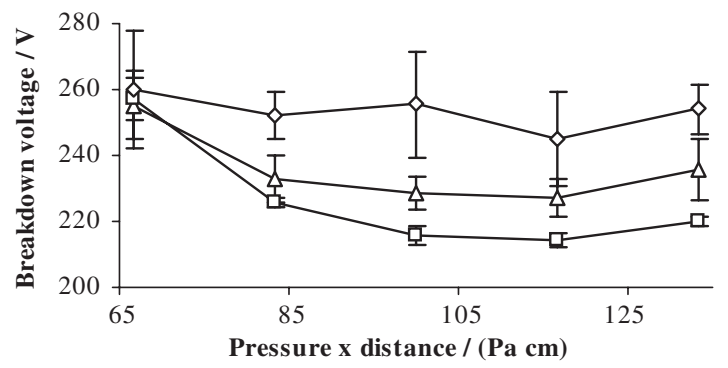

Figure 3. Breakdown voltages measured at three different electrode distances $(\triangle, 0.01 \mathrm{~cm}, \square, 0.025 \mathrm{~cm}, \diamond, 0.05 \mathrm{~cm})$. Pressure values were different for each electrode distance to allow for comparison of same pressure $x$ distance product. region (between 100 and $120 \mathrm{Pacm}$ ). It has to be noted that the data sets are not in the order of gap distance, i.e. the lowest breakdown voltages have been measured for the $0.025 \mathrm{~cm}$ gap and not for the $0.01 \mathrm{~cm}$ (or $0.05 \mathrm{~cm}$ ). Higher values of standard deviations have been observed for the breakdown curve with higher voltages $(0.05 \mathrm{~cm})$; measurements for the $0.05 \mathrm{~cm}$ gap show a maximum relative standard deviation of $7 \%$, while the $0.01 \mathrm{~cm}$ curve shows a maximum of $4.1 \%$ and the $0.025 \mathrm{~cm}$ presents the lowest variation with $2.5 \%$ relative standard deviation (i.e. in order of breakdown voltage values).

The variations in breakdown voltage for each of the curves have to be attributed mainly to changes in cathode properties and cleanliness (Jacobs and La Roque 1947, Pace and Parker 1973, Phelps and Petrovic 1999). Nevertheless, they can be considered very small, in particular for the $0.025 \mathrm{~cm}$ gap distance. This has to be appreciated in consideration of the fact that the measurements were performed for dirty surfaces; it seems that the breakdown voltages are repeatable within the same device.

On the other hand, the differences between the three gap distances can find justification in several phenomena (Auday et al 1998). It could be suggested that different electron emission phenomena have taken place in the three cases. Unfortunately, if this were true, more and different experimental results would be needed. It is also very likely that the reproducibility of the device has had a large influence and it had determined the diversity of measurements. The poorer reproducibility of the device, compared with the repeatability of the measurements, can be attributed to moisture, epoxy fumes, spacing accuracy, etc. After all, pixels within the same device that had been exposed to very similar surface conditions and manufacturing procedure did show better repeatability of results.

With these considerations we would be inclined to attribute voltage variations to differences between electrode surfaces and device manufacturing processes; note that within the same device, breakdown measurement fluctuations could be contained. This does not exclude the real possibility that electrode distance alone affects breakdown, but this hypothesis would need further and different investigations.

\subsection{Secondary electron emission coefficient}

The secondary electron emission coefficient provides information on the efficiency of electron emission from the cathode due to ion bombardment, and it is normally represented by the Greek letter $\gamma$. There are methods and models that allow the evaluation of this coefficient from experimental data under the hypothesis that electron emission by ions is the only means of electron production from the cathode (Pace and Parker 1973, Phelps and Petrovic 1999, Auday et al 2000). Emission by ions can be one of the major causes of electron emission, but it is never the only cause and often it is not dominant. Nevertheless, it is normal practice to proceed with the evaluation of the secondary electron emission and rename the coefficient obtained an effective secondary electron emission coefficient (Auday et al 1998, 2000, Phelps and Petrovic 1999, Phelps et al 1999). We therefore adopt this convention in our evaluation, and throughout this paper we will refer to the effective coefficient, even when not explicitly written. 
In this paper, in order to determine the effective coefficients, we will utilize the breakdown voltage measurements of the proceeding section (Petrovic and Phelps 1997, Auday et al 1998, Phelps and Petrovic 1999). The theory for this calculation is based on $\gamma$ and on the primary ionization coefficient, $\alpha$, where the latter expresses the number of single ionizing collisions by an electron in unit distance. The two coefficients both appear in the self-sustaining condition for a homogeneous electric field (Phelps and Petrovic 1999, Braithwaite 2000),

$$
\alpha d=\ln \left(1+\frac{1}{\gamma}\right) .
$$

The primary coefficient assumes validity when equilibrium conditions for electron drift velocity are reached (Pace and Parker 1973, Phelps et al 1993, Braithwaite 2000). These would not normally be satisfied on the left-hand side of the Paschen minimum, but in this particular case, it is noted that the electric field at and before breakdown is not excessively high (Pace and Parker 1973). It has been reported that the electron drift velocity can be considered to be in equilibrium for $E / n$ (electric field to density ratio, where $n$ is the gas density) below or in the range of $10^{-18} \mathrm{~V} \mathrm{~m}^{2}$; the range of $E / n$ used for the results reported in this paper satisfies this condition, and it is therefore assumed that the use of $\alpha$ is meaningful (Pace and Parker 1973). Given the validity of $\alpha$, this coefficient can be expressed in a parametrized form,

$$
\alpha=A p \exp \left(\frac{-B p}{E}\right)
$$

where $A$ and $B$ are constants of the gas being used; for argon these are $A=0.09 \mathrm{~Pa}^{-1} \mathrm{~cm}^{-1}$ and $B=1.35 \mathrm{~V} \mathrm{~Pa}^{-1} \mathrm{~cm}^{-1}$ (Raizer 1991, Braithwaite 2000). The pressure, $p$, is obtained from the gas density by assuming an ambient temperature of 298.16 K. This expression for the primary coefficient loses validity for higher values of the ratio of the electric field to density, and more recently empirical fits or results of numerical models have been given (Phelps et al 1993, Petrovic and Phelps 1997, Auday et al 1998, 2000, Phelps and Petrovic 1999). Nevertheless, for the range of $E / n$ used here (growing part of $\alpha$ ), equation (2) is a good representation of the ionization coefficient; the differences with the empirical fit of Phelps and Petrovic (1999) were less than 5\%.

Combining equation (1) with (2) and considering that before and at breakdown the electric field is uniform along the discharge axis (i.e. $E=V / d, V$ is the electrode voltage), we obtain

$$
\gamma=\frac{1}{\mathrm{e}^{\alpha d}-1}=\frac{1}{\exp (A p d \cdot \exp (-B p d / V))-1} .
$$

Equation (3) can then be used to determine the secondary electron emission coefficient from breakdown voltage measurements.

Results for the secondary electron emission coefficient against the ratio of the electric field to density are shown in figure 4. These were determined using equation (3), where $V$ was obtained from breakdown voltage measurements at the specified values of pressure $(p)$ and distance $(d)$. The values of the constants $A$ and $B$ are those for argon as mentioned above. Three data sets corresponding to the three

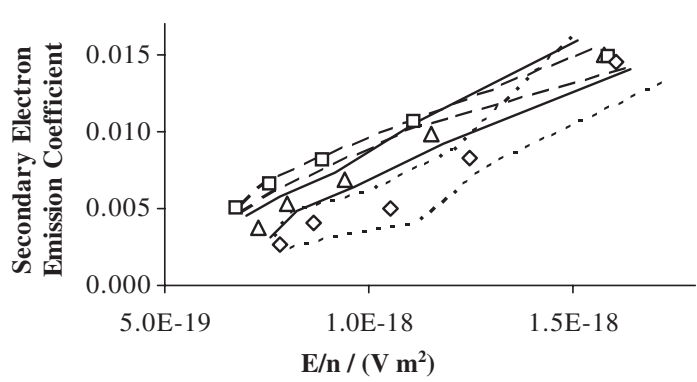

Figure 4. Secondary electron emission coefficient as calculated from measured breakdown voltage for three different electrode distances $(\triangle, 0.01 \mathrm{~cm}, \square, 0.025 \mathrm{~cm}, \diamond, 0.05 \mathrm{~cm})$. Each set of experimental data also has contour lines indicating the variations introduced by the standard deviations of the breakdown voltage measurements (full curves, $0.010 \mathrm{~cm}$, broken curves, $0.025 \mathrm{~cm}$ and dotted curves, $0.050 \mathrm{~cm}$ ).

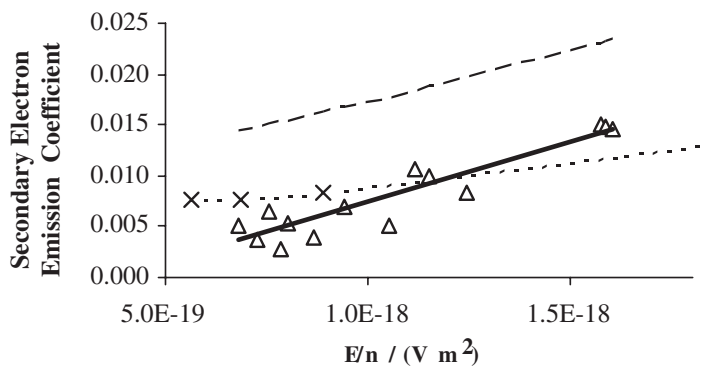

Figure 5. Comparison of secondary electron emission coefficients: experimental results with tin oxide electrodes from this paper $(\triangle)$ and showing a linear fit (thick line) through all data points; results for gold-plated copper electrodes from Petrovic and Phelps (1997, broken curve) and copper electrodes from Auday et al (1998, $X$ connected with dotted lines).

electrode distances are shown; for each data set, contour lines are depicted illustrating the variations introduced by the breakdown voltage standard deviations. As for the breakdown voltage measurements, three similar but distinct behaviours can be observed.

The comments related to the differences in $\gamma$ values among the three data sets arise from the breakdown voltage, so that differences in cathode surfaces, influence of different electron emission processes, repeatability of measurements and reproducibility of devices can all apply to these results. More interesting is the general increasing linear trend with the ratio of the electric field to density, which agrees with other results for different electrode materials and for different combinations of the product $p d$ (Phelps and Petrovic 1999). It has to be noted that linearity is probably mainly due to the small range of the ratio of the electric field to density that has been studied here.

In figure 5 the results of this paper are reported in comparison with other results obtained for argon discharges with a copper electrode and gold-plated copper (Petrovic and Phelps 1997, Auday et al 1998); a linear fit to our experimental data is also shown $\left(\gamma=1.2 \times 10^{16} \times E / n-0.0043\right)$. The results obtained with a gold-plated copper electrode are represented by an approximated formula (Petrovic and Phelps 1997), while the data for the copper electrode are given in tabulated values (Auday et al 1998). The increase in the coefficient is evident and very similar in all three cases, while 


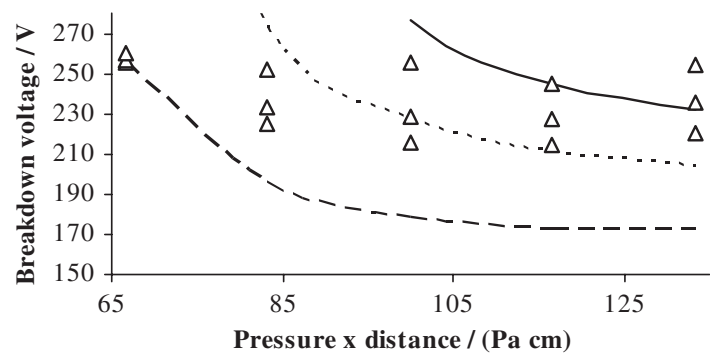

Figure 6. Breakdown curves from equation (4) for different constant values of $\gamma$ (full curve for $\gamma=0.004$, dotted curve for $\gamma=0.007$ and broken curve for $\gamma=0.015$ ) and experimental results of breakdown voltage in this paper $(\triangle)$.

the gold-plated electrodes show a higher electron emission efficiency. Comparison with the review article of Phelps and Petrovic (1999) shows a general agreement in terms of the growth with the ratio of the electric field to density; as no other results are reported for tin oxide, differences are expected and add importance to the results shown here. Tin oxide seems to have lower values of the secondary electron emission coefficient, but it presents a steeper growth and $\gamma$ reaches values comparable with other metallic surfaces when higher $E / n$ values are approached. In Auday et al (2000), secondary electron emission coefficients for dielectric material used in plasma displays have been reported. Unfortunately the coefficients have been calculated for a lower range of $E / n$, although it seems obvious the coefficient is higher for $\mathrm{MgO}$ with respect to tin oxide by one order of magnitude.

\subsection{Effect of secondary electron emission coefficient on a form of breakdown law}

A widely accepted form of breakdown law is the following:

$$
V=\frac{B p d}{\ln (A p d)-\ln \left(\ln \left(1+1 / \gamma_{i}\right)\right)},
$$

where $V$ is the breakdown voltage, $A$ and $B$ are constants of the gas being used and $\gamma_{i}$ is the ion induced secondary electron emission coefficient (Braithwaite 2000, Hartherz et al 2000, Miller 1964). Equation (4) can be used to produce a curve of theoretical breakdown voltages and it is valid where electron production at the surface is dominated by ion collisions.

Figure 6 compares experimental breakdown voltages with voltage values calculated with equation (4), for which three different constant values of $\gamma_{i}$ have been used $(0.004,0.007$ and 0.015). As can be noted, equation (4) does not represent experimental data, well and this deviation from theory has been reported previously (Hartherz et al 2000, Lisovsky and Yakovin 2000).

There are several factors that may contribute to the deviations between the experimental results and the theoretical prediction of equation (4). The ratio between the electrode distance, $d$, and the electrode radius, $R$, has been shown to have an influence on the breakdown law (Lisovskiy and Yakovin 2000 , Lisovskiy et al 2000). In this case, the ratio $d / R$ was between about 0.03 and 0.17 , and it could have affected the breakdown at some extent, but the absence of a surrounding wall for each pixel of the micro-plasma device makes this possibility less likely. More important for this study is that

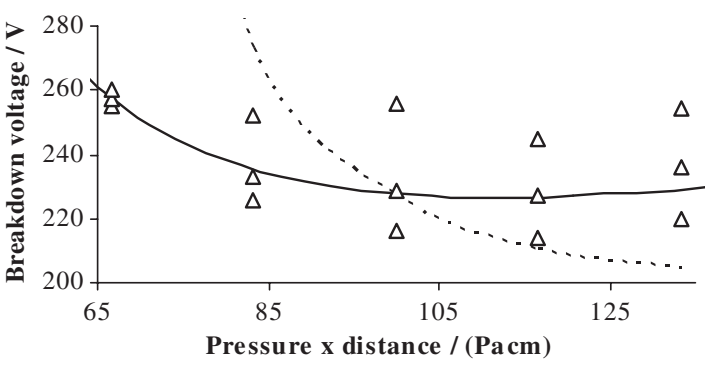

Figure 7. Experimental breakdown measurements obtained in this study $(\triangle)$, breakdown curve from equation (4) for $\gamma=0.007$ (dotted curve) and again equation (4) where $\gamma$ has been considered a linear variation of the electric field to density ratio (full curve).

at these conditions of pressure and distance, ions are not the only cause of electron emission and that other phenomena of electron production need to be accounted for (Phelps et al 1993, Phelps and Petrovic 1999, Auday et al 2000). In agreement with the convention of using an effective secondary electron emission coefficient, it could be suggested that this could replace the ion induced electron emission coefficient, $\gamma_{i}$, in equation (4). Nevertheless the changes of $\gamma$ with $E / n$ that have been discussed in the previous sections will have to be taken in consideration. This substitution leads to an implicit form of equation (4) that can be solved numerically.

Figure 7 shows the results obtained when substituting $\gamma_{i}$ in equation (4) with the linear relation of the effective coefficient determined in the previous section. Although figure 7 mainly reinstates the validity of a linear growth for the effective coefficient in the range of $E / n$ studied here, it also suggests the possibility of using effective coefficient data for the prediction of breakdown voltage as a correction factor for the unaccounted electron production phenomena (Jacobs and La Roque 1947).

\section{Conclusion}

We have presented breakdown voltage measurements for a range of pressure and distance that is of interest for different applications. These results were obtained with tin oxide electrodes; tin oxide is a material that offers useful optical properties and is widely used for plasma displays. On the other hand, there are few published results for these conditions, which adds importance to our results.

We then proceeded with determination of the effective secondary electron emission coefficient from breakdown experimental data. These results are in agreement with other results obtained for other electrode materials; for the small range of $E / n$ studied here, a linear growth was observed. Data on the secondary electron emission coefficient are essential to numerical modelling, and we intend to use these findings in the development of a model for the discharges produced with this micro-plasma device. This will contribute in obtaining electron/ion density and electric field strength distribution.

A comparison with a form of breakdown law revealed that the experimental breakdown voltages deviates from theoretical prediction. This has suggested that other electron production phenomena have a noticeable effect on the generation and maintenance of the discharge.

Further experimental investigations need to be done in order to assess the influence of the distance alone on 
the breakdown measurements. Also, the reproducibility of the micro-plasma device needs to be addressed for both applicability of the device in gas analysis and for a more in-depth understanding of the influence of single parameters.

\section{References}

Auday G, Guillot Ph and Galy J 2000 Secondary emission of dielectrics used in plasma display panels J. Appl. Phys. $\mathbf{8 8}$ 4871-4

Auday G, Guillot Ph, Galy J and Brunet H 1998 Experimental study of the effective secondary emission coefficient for rare gases and copper electrodes J. Appl. Phys. 83 5917-21

Braithwaite N St J 2000 Introduction to gas discharges Plasma Sources Sci. Technol. 9 517-27

Hartherz P, Yahia K B, Subert C, Mueller L, Pfendtner R, Hiller W and Pfeiffer W 2000 Experimental investigation of breakdown voltages for micrometer gaps-deviations from Paschen's law Proc. International Conf. on Gas Discharges and their Applications vol 1, pp 493-6

Hyland M, Mariotti D, Dubitzky W, McLaughlin J A and Maguire P 2000 Gas recognition using a neural network approach to plasma optical emission spectroscopy Proc. SPIE-Int. Symp. on Opt. Sci. 4120 246-52

Jacobs H and La Rocque A P 1947 Minimum sparking potentials of barium, magnesium, and aluminum in argon J. Appl. Phys. 16 199-203

Jelenak Z M, Velikic Z B, Bozin J V, Petrovic Z Lj and Jelenkovic B M 1993 Electronic excitation of the 750- and 811-nm lines of argon Phys. Rev. E 47 3566-73

Lemoine P, Mariotti D, Maguire P and McLaughlin J A 2001

Depth sensitive analysis of a degraded tin oxide electrode surface in a plasma device application Thin Solid Films $\mathbf{4 0 1}$ 196-202
Lisovskiy V A and Yakovin S D 2000 A modified Paschen law for the initiation of a DC glow discharge in inert gases Tech. Phys. 45 727-31

Lisovskiy V A, Yakovin S D and Yegerenkov V D 2000 Low-pressure gas breakdown in uniform dc electric field J. Phys. D: Appl. Phys. 33 2722-30

Mariotti D, Shannon J, Schreitmueller H and McLaughlin J 2000 A technique to use gas discharge spectroscopy for artificial nose applications Proc. International Conf. on Gas Discharges and their Applications vol 2, pp 760-3

Miller H C 1964 Breakdown potential of neon below the Paschen minimum Physica 30 2059-67

Novak J P and Bartnikas R 2000 Effect of dielectric surfaces on the nature of partial discharges IEEE Trans. Diel. Electr. Insul. 7 $146-51$

Pace J D and Parker A B 1973 The breakdown of argon at low pressure J. Phys. D: Appl. Phys. 6 1525-36

Petrovic Z Lj and Phelps A V 1997 Temporal and constriction behaviour of low-pressure, cathode dominated argon discharges Phys. Rev. E 56 5920-31

Phelps A V and Petrovic Z Lj 1999 Cold-cathode discharges and breakdown in argon: surface and gas phase production of secondary electrons Plasma Sources Sci. Technol. 8 R21-44

Phelps A V, Petrovic Z Lj and Jelenkovic B M 1993 Oscillations of low-current electrical dsicharges between parallel-plane electrodes: III. Models Phys. Rev. E 47 2825-38

Phelps A V, Pitchford L C, Pedoussat C and Donko Z 1999 Use of secondary-electron yields determined from breakdown data in cathode-fall models for Ar Plasma Sources Sci. Technol. 8 B1-2

Raizer Y P 1991 Gas Discharge Physics (Berlin: Springer)

Stefanovic I and Petrovic Z L 1997 Volt ampere characteristics of low current DC discharge in $\mathrm{Ar}, \mathrm{H}_{2}, \mathrm{CH}_{4}$ and $\mathrm{SF}_{6}$ Japan. $J$. Appl. Phys. 36 4728-32 